Int. J. Dev. Biol. 55: 109-119 (2011)

doi: $10.1387 / \mathrm{ijdb} .103109 \mathrm{rk}$

\title{
Targeted expression of p35 reveals a role for caspases in formation of the adult abdominal cuticle in Drosophila
}

\author{
RACHEL S. KESTER and JOHN R. NAMBU* \\ Department of Biology and Molecular and Cell Biology Graduate Program, \\ University of Massachusetts at Amherst, Amherst MA, USA
}

\begin{abstract}
Programmed cell death is a fundamental aspect of metazoan development associated with the elaboration of disparate tissues and structures. Specialized cysteine proteases, the caspases, are mediators of cell death; once activated they cleave substrate proteins to dismantle doomed cells. Caspase activity is regulated by several cellular and viral inhibitors. The baculovirus p35 protein blocks the action of a wide range of caspases and inhibits cell death in divergent species. Here, we utilize the Gal4/UAS system to target p35 expression and analyze the requirements of caspase activity for development in Drosophila. We confirm that cell death is essential for proper morphogenesis of the adult male external genitalia and distal portions of the legs. In addition, we find that caspases are also required for elimination of larval epidermal cells and normal elaboration of the adult abdominal cuticle by histoblast derivatives. In particular, rescued p35-expressing larval epidermal cells accumulate along the abdominal midline and are associated with corresponding splits in both dorsal and ventral cuticle structures. This study reveals a novel role for cell death in a specific morphogenetic processes.
\end{abstract}

KEY WORDS: Drosophila, caspases, p35, cell death, morphogenesis, abdomen

During animal development, programmed cell death eliminates many different cell types and tissues that are present in excess number, become damaged or infected, or have transient functions (reviewed in Domingos and Steller 2007; Baehrecke 2002; Meier et al. 2000). The importance of cell death in development and physiological homeostasis is demonstrated by the pathology associated with disruptions in normal cell death patterns and levels. Either insufficient or excessive cell death can have very detrimental effects on human health and may result in congenital malformations, cancer, autoimmune disorders, and neurodegenerative diseases.

Programmed cell death can be driven by several distinct processes, including apoptosis, autophagy, and necroptosis, that are each associated with specific biochemical and cellular properties (reviewed in Christofferson and Yuan 2009; Hotchkiss et al. 2009; Krysko et al. 2008). These death processes often involve the actions of specialized cysteine proteases, the caspases (reviewed in Chowdhury et al. 2008; Salvesen and Riedl 2008). Caspases are highly conserved and cleave substrate proteins at Asp or Glu residues in the context of specific recognition se- quences. They are initially synthesized as inactive zymogens that require internal cleavage to liberate two subunits that assemble into functional heterotetramers. Initiator caspases contain extended prodomains that regulate their dimerization and activation in response to pro-death signals. This regulation is afforded by controlled incorporation into multi-protein complexes such as the apoptosome or Death Inducing Signaling Complex (DISC) that mediate intrinsic and extrinsic pathways of apoptosis (reviewed in Taylor et al. 2008). Once activated, initiator caspases cleave and activate effector caspases that contain smaller pro-domains and ultimately carry out the orderly dismantling of the cell. In $C$. elegans, the Ced-3 caspase is necessary and sufficient for all developmental cell deaths (Yuan et al. 1993). In contrast, both flies and humans possess multiple initiator and effector caspases

\footnotetext{
Abbreviations used in this paper: ADN, anterior dorsal nest; APF, after puparium formation; GFP, green fluorescent protein; IAP, inhibitor of apoptosis protein; LEC, larval epidermal cells; NLS, nuclear localization signal; PDN, posterior dorsal nest; $\mathrm{SN}$, spiracular nest; VN, ventral nest.
}

\footnotetext{
*Address correspondence to: John R. Nambu. Department of Biological Sciences, Florida Atlantic University, Boca Raton FL 33431, USA. Fax: +1-561-297-2749. e-mail: jnambu@fau.edu
} 
(reviewed in Hay and Guo 2006; Riedl and Shi 2004). In addition to their roles in programmed cell death, caspases have proteolytic processing activities important for lymphokine activation and immune response, transcription factor processing, cell migration, and spermatid individualization (reviewed in Feinstein-Rotkopf and Arama 2009; Li and Yuan 2008; Lamkanfi et al. 2007). It is not yet fully understood how caspases that activate programmed cell death are also able to mediate other processes without eliciting the demise of the cell; however, it is likely that their levels, substrates, or sites of activity are differentially regulated.

Given the important and diverse functions of caspases, it is not surprising that their activities are tightly controlled. Many viruses express caspase inhibitors that facilitate their propagation by blocking the ability of infected host cells to undergo cell death. Significantly, two important types of caspase inhibitors have been identified from insect baculoviruses, including Inhibitor of Apoptosis proteins (IAPs) and p35 (reviewed in Clem 2007). Both cellular and viral IAPs have been characterized; they bind caspases to prevent their activation or activity (reviewed in Mace et al. 2010; Orme and Meier 2009; Steller 2008). IAPs may also promote caspase ubiquitination and degradation. The structurally unrelated caspase inhibitor, p35, acts as a poison substrate; it is cleaved and forms a thioester bond with the caspase active site, thereby causing irreversible inhibition of the enzyme (Xu et al. 2003; 2001; dela Cruz 2001). Unlike IAPs, p35 is not well conserved and no cellular counterparts have been identified. Nonetheless, p35 effectively inhibits a broad range of active caspases and can block cell death not only in insects, but also in C. elegans, vertebrates, and plants (Danon et al. 2004; Izquierdo et al. 1999; Xue and Horvitz 1995; Sugimoto et al. 1994). In Drosophila, targeted expression of p35 blocks both naturally occurring developmental cell death as well as ectopic cell death induced by genetic or environmental means (Davidson and Steller 1998; Hay et al. 1994).

One particularly striking example of programmed cell death during development is the coordinated destruction of the larval form of organisms that undergo complete metamorphosis. In this regard, Drosophila is holometabolous and while both the wormlike larva and hexapod adult are motile and have parallel feeding and sensory capabilities, the adult fly is the reproductive stage and possesses highly specialized locomotor and sensory appendages. These structures arise during pupation in a process associated with the degeneration of many larval tissues, including components of the nervous system, musculature, salivary glands, and digestive system. Formation of the adult form thus requires coordination of extensive cell destruction, proliferation, and differentiation processes. During this process, the entire adult body becomes covered by a tough chitin-based cuticle, which provides both structural and protective functions. As the adult form is delimited by this exoskeleton, proper formation of cuticular structures is crucial to the elaboration of the adult morphology.

The cells that give rise to the adult cuticle derive from two major sources: imaginal discs and histoblast nests (reviewed in Fristrom and Fristrom 1993). These groups of epithelial cells are specified during embryogenesis and proliferate and differentiate during pupation. Imaginal disc derivatives generate the head capsule and thorax, including all the appendages, as well as the external genitalia and anal plates. Histoblast progeny generate most of the abdominal cuticle. There are four pairs of histoblast nests present in each segment of the abdomen (Madhavan and Schneiderman 1977), including the anterior dorsal nest (ADN), the posterior dorsal nest (PDN), the ventral nest (VN), and the spiracular nest (SN). At the onset of pupation, 0 hours after puparium formation (APF), histoblast nests are small, and each contain only 6-14 cells. Within 2-4 hours APF, histoblast cells begin to divide rapidly and by 4-8 hours APF both ADNs and PDNs contain approximately 250 cells that accumulate in columns. Once histoblast nests have expanded, the migration of histoblast cells is initiated. By 18-20 hours APF, the larval epidermal cells (LECs) between the ADNs and PDNs begin to disappear as fusion of the two segmental nests begins. During this time, cells of the VN and SN also migrate towards each other and fuse. At 30-36 hours APF, the histoblast cells on the dorsal and ventral lateral sides fuse and by 41 hours APF, both lateral sides have fused at the dorsal and ventral midlines. 48 hours APF, fusion is complete and LECs are no longer detected. The histoblast cells begin to secrete the adult cuticle. On the dorsal side of the abdomen this includes a series of hard segmentally repeated tergite plates separated by soft, flexible intersegmental membrane. On the ventral side, there is a soft pleurite cuticle with small circular sternite pads along the midline. A stereotypic pattern of bristles grows out of each tergite and sternite. By 72 hours APF, the bristles and hair are completely formed and become pigmented at 90 hours APF. At 96 hours APF, metamorphosis is essentially complete and the adult fly is ready to emerge (Madhavan and Madhavan 1980). While it is not entirely clear how the histoblasts replace the LECs during this process, both ablation experiments and high resolution cellular analyses suggest that the histoblasts may provide signals that promote elimination of the LECs (Madhavan and Madhavan 1984; 1980; Ninov et al. 2010; 2007).

Given the strong conservation of programmed cell death processes, it is somewhat surprising that cell death is not necessarily essential for viability or development in all metazoans. Thus, in C. elegans where approximately $12 \%$ of all somatic cells normally die (reviewed in Lettre and Hengartner 2006), mutant worms lacking all cell death develop into viable and fertile adults, despite the presence of many ectopic cells (Ellis and Horvitz 1986). In mammals, cell death does appear to be essential for development as some caspase knockout mutant mice exhibit lethality that is associated with significant brain hypertrophy or other tissue abnormalities (reviewed in Galluzzi et al. 2008; Wang and Lenardo 2000; Zheng et al. 1999). In Drosophila, the situation is less clear. $\mathrm{Df}(3 \mathrm{~L}) \mathrm{H} 99$ mutants lack cell death and exhibit embryonic lethality, but this deletion removes multiple genes that may be essential for viability (reviewed in Steller 2008; Bergmann etal.2003). Mutations in the apical caspse droncresult in a severe reduction in developmental cell death, but even dronc null mutants are not completely lethal (Xu et al. 2005; Daish et al. 2004; Chew et al. 2004). Significantly, there exist effective compensatory proliferation mechanisms that facilitate normal development despite significant disruptions in cell death patterns or abnormal cell population sizes (reviewed in Martin et al. 2009; Fan and Bergmann 2008)

In this study we address the question of which tissues require caspase activities for viability and normal development in Drosophila. We undertook a small scale genetic screen using 32 different $\mathrm{P}[\mathrm{Gal} 4]$ lines and P[UAS-p35] to disrupt caspase activities in a wide range of developing cells and tissues. We find that 
inhibition of caspases in many distinct tissues does not result in lethality or anatomical malformations; p35 expression from the majority of $P[G$ al4] lines analyzed did not result in any detectable phenotype. As expected, P[Gal4] lines were identified that produced morphological disruptions in the male external genitalia as well as elaboration of distal leg segments. Strikingly, several $P[G a 14]$ lines that drove p35 expression in LECs were found to result in semi-lethal phenotypes where the surviving flies exhibited severe disruptions in the adult abdominal cuticle. In particular, there were large midline splits along the dorsal cuticle where the tergites failed to fully extend and fuse as well as similar disruptions along the midline of the ventral sternites. These defects were associated with disruption in the normal death of the LECs, as ectopic surviving LECs accumulated along the midline. These studies thus identify a novel morphogenetic process that requires caspase activities and provide new insight into the developmental requirements for programmed cell death.

\section{Results}

Targeted p35 expression disrupts adult abdomen formation A small genetic screen was performed to identify tissues that require caspase activities for normal development. 32 different $\mathrm{P}$ [Gal4] lines (see Materials and Methods) were used to drive expression of the p35 caspase inhibitor in a wide array of developing tissues, including mesodermal (e.g. P[Gal4-twi.G]2.2), ectodermal (e.g. P[Gal4-prd.F]RG1), and endodermal (e.g. P[drmGal4.7.1]1.1) derivatives (Table 1). The sites of expression for these P[Gal4] include the central and peripheral nervous system, skeletal and visceral musculature, epidermis, foregut, midgut, hindgut, salivary glands, reproductive tissues and imaginal discs in embryonic, larval, or pupal stages (http:// flystocks.bio.indiana.edu/Browse/misc-browse/gal4.htm). Notably, the expression patterns listed for these lines are not comprehensive and therefore correspond to the minimal sites of expres-

TABLE 1

\section{P35 MIS-EXPRESSION SCREEN}

\begin{tabular}{|c|c|c|}
\hline GAL4 Driver & Phenotype with P35 at $25^{\circ} \mathrm{C}$ & Expression Pattern \\
\hline $\mathrm{P}\{\mathrm{GawB}\} 32 \mathrm{~B}$ & Lethal, abdomen cuticle defects & Expressed in imaginal discs \\
\hline$P\{G a w B\} 645 b$ & Semi-lethal, abdomen cuticle defects & Expressed in pattern in oocyte: posterior pole \\
\hline P\{Ddc-GAL4.L\}4.3D & Semi-lethal, abdomen cuticle defects & Expressed in dopaminergic and serotonergic neurons \\
\hline P\{Eip71CD-GAL4.PC\}TP1-1 & Semi-lethal, abdomen cuticle defects & Expressed in the larval epidermis and in brain cells starting at the mid-third instar transition \\
\hline$P\{G a w B\} 34 B$ & Semi-lethal, abdomen cuticle defects & Expressed in embryonic salivary glands, posterior midgut, eye-antennal, haltere, leg and wing imaginal discs \\
\hline$P\{G a w B\} 389$ & Semi-lethal, abdomen cuticle defects & Expressed in embryonic CNS \\
\hline$P\{G a w B\} 71 B$ & Semi-lethal, abdomen cuticle defects & Expressed in imaginal discs \\
\hline$P\{G a w B\} 109-69$ & Semi-lethal abdomen cuticle defects & $\begin{array}{l}\text { Expressed in embryonic dorsal epidermis and in parts of the peripheral nervous system, in the larval wing imaginal disk and } \\
\text { parts of the brain, and in stage } 8 \text { follicle cells }\end{array}$ \\
\hline$P\{G a w B\} c 179$ & Semi-lethal, abdomen cuticle defects & Expressed in embryonic mesoderm, larval muscles and wing imaginal discs \\
\hline$P\{$ GawB $\}$ T80 & Semi-lethal, abdomen cuticle defects & Expressed ubiquitously in third instar imaginal discs \\
\hline$P\{$ drm-GAL4.7.1\}1.1 & Semi-lethal male genital defects & Expressed in embryonic proventriculus, anterior midgut, posterior midgut, Malpighian tubules, and small intestine \\
\hline $\mathrm{P}\{$ GawB $\} 227$ & Semi-lethal male genital defects & Expressed in epidermis and CNS starting at embryonic stage 10 \\
\hline$P\{G a w B\} D \|[m d 23]$ & Semi-lethal adult leg truncations & Expressed in DII pattern \\
\hline$P\{G a w B\} 109-30$ & Normal & $\begin{array}{l}\text { Expressed in the embryonic dorsal midline and PNS, the larval ventral nerve cord and segmentally in the nervous system, } \\
\text { and in stalk cells, follicle cells at the poles of the egg chamber and the precursors to the follicle cells in the germarium }\end{array}$ \\
\hline P\{GAL4-Mef2.R\}3 & Normal & Expressed in muscle cells \\
\hline$P\{G a w B\} c 81$ & Normal & Expressed in third instar larva: brain - strong throughout CNS \\
\hline$P\{G a w B\} 4 G$ & Normal & $\begin{array}{l}\text { Expressed in pan-neural in late embryos, in a subset of motor neurons in 3rd instar larvae, and enriched in mushroom } \\
\text { bodies in adults }\end{array}$ \\
\hline $\mathrm{P}\{\mathrm{GawB}\} \mathrm{c} 135$ & Normal & $\begin{array}{l}\text { Expressed in embryonic proventriculus, larval brain, eye disc, gut, fat body, adult ovarian squamous and post. terminal } \\
\text { follicle cells, male access. glands, seminal vesicles, ejaculatory duct, cyst cells and spermatocytes }\end{array}$ \\
\hline$P\{d p p-G A L 4 . P S\} 6 A$ & Normal & $\begin{array}{l}\text { Expressed in spiracular branch } 10 \text { and posterior spiracle beginning at stage } 12 \text {, continuing through stage } 17 \text {, and cells of the } \\
\text { dorsal ectoderm }\end{array}$ \\
\hline P\{GAL4\}repo & Normal & Expressed in glia \\
\hline$P\{$ GawB $\} 167 Y$ & Normal & $\begin{array}{l}\text { Expressed in third instar larva: brain - neuroblasts in central brain and ventral ganglion, Bolwig's nerve, discs - spot in leg } \\
\text { disc only }\end{array}$ \\
\hline$P\{$ GawB\}elav[C155] & Normal & Expressed in all tissues of the embryonic nervous system beginning at stage 12 \\
\hline$P\{G a w B\} \mid(2) C 805[C 805]$ & Normal & $\begin{array}{l}\text { Expressed in larval ring gland, histoblasts, gut and Malpighian tubules and adult male accessory glands, testis sheath and } \\
\text { cyst cells }\end{array}$ \\
\hline$P\{G a w B\} \mid(3) 31-1[31-1]$ & Normal & Expressed in neuroblasts and neurons \\
\hline P\{Hml-GAL4.G\}5-6 & Normal & Expressed in larval hemocytes; hml normally expressed at low levels \\
\hline P\{GAL4-twi.G\}2.2 & Normal & Expressed in a broad mesodermal swath during embryonic stage 8 \\
\hline P\{GAL4-ninaE.GMR\}12 & Normal & Expressed in the eye disc, provides strong expression in all cells behind the morphogenetic furrow \\
\hline$P\{G a w B\} 109 C 1$ & Normal & Expressed in the embryonic epidermis and in the follicle cells at the anterior and posterior poles of the egg chamber \\
\hline P\{EcR.GET-BD-GAL4\}1 & Normal & Expressed in larval EcR-A-expressing neurons destined for apoptosis at metamorphosis; also expressed in imaginal discs \\
\hline P $\{$ en2.4-GAL4\}e22c & Normal & Expressed ubiquitously in embryo \\
\hline$P\{G a w B\} b a b 1[$ Agal4-5] & Normal & $\begin{array}{l}\text { Expressed in ovarian terminal filament, testes, haltere, antennal, female genital and wing discs, tarsal segments of leg } \\
\text { disc,and embryonic posterior spiracles, salivary glands and proventriculus }\end{array}$ \\
\hline $\mathrm{P}\{$ GawB $\}$ c309 & Normal & Expressed in mushroom body, thoracic ganglion and eye-antennal disc \\
\hline P\{GAL4-prd.F\}RG1 & Normal & Expressed in prd pattern \\
\hline$P\{G a w B\} 52 a$ & Normal & Expressed in CNS midline \\
\hline
\end{tabular}


sion. The progeny resulting from crosses between $\mathrm{P}[\mathrm{Gal} 4]$ and P[UAS-p35] lines were classified into two major categories: 1) fully viable with no overt phenotype, or 2) lethal or semi-lethal with morphological abnormalities.

The first category included the majority of the P[Gal4] lines analyzed. Thus, 21 of the 32 lines tested yielded fully viable progeny flies that did not display any morphological or anatomical abnormality. Interestingly, among these were a number of lines that drive expression in tissues where cell death is prominent, including the CNS midline (e.g. P[GawB]52a), embryonic epidermis (e.g. P[Gal4-prd.F]RG1]), imaginal discs (e.g. P[GawB]T80), and glia (e.g. P[repo-Gal4]). Full viability was also observed for drivers that yield widespread or ubiquitous expression (e.g. $\mathrm{P}$ [en2.4Gal4]e22c). The second category included $11 \mathrm{P}[\mathrm{Gal} 4]$ lines where the p35-expressing progeny exhibited lethality or semi-lethality and adult anatomical defects. Within this category, 9 P[Gal4] lines (e.g. P[GawB]32B, P[GawB]645b, P[ddcGal4.L]4.3D, P[Eip71CD-Gal4.PC], P[GawB]34B, P[GawB]389,
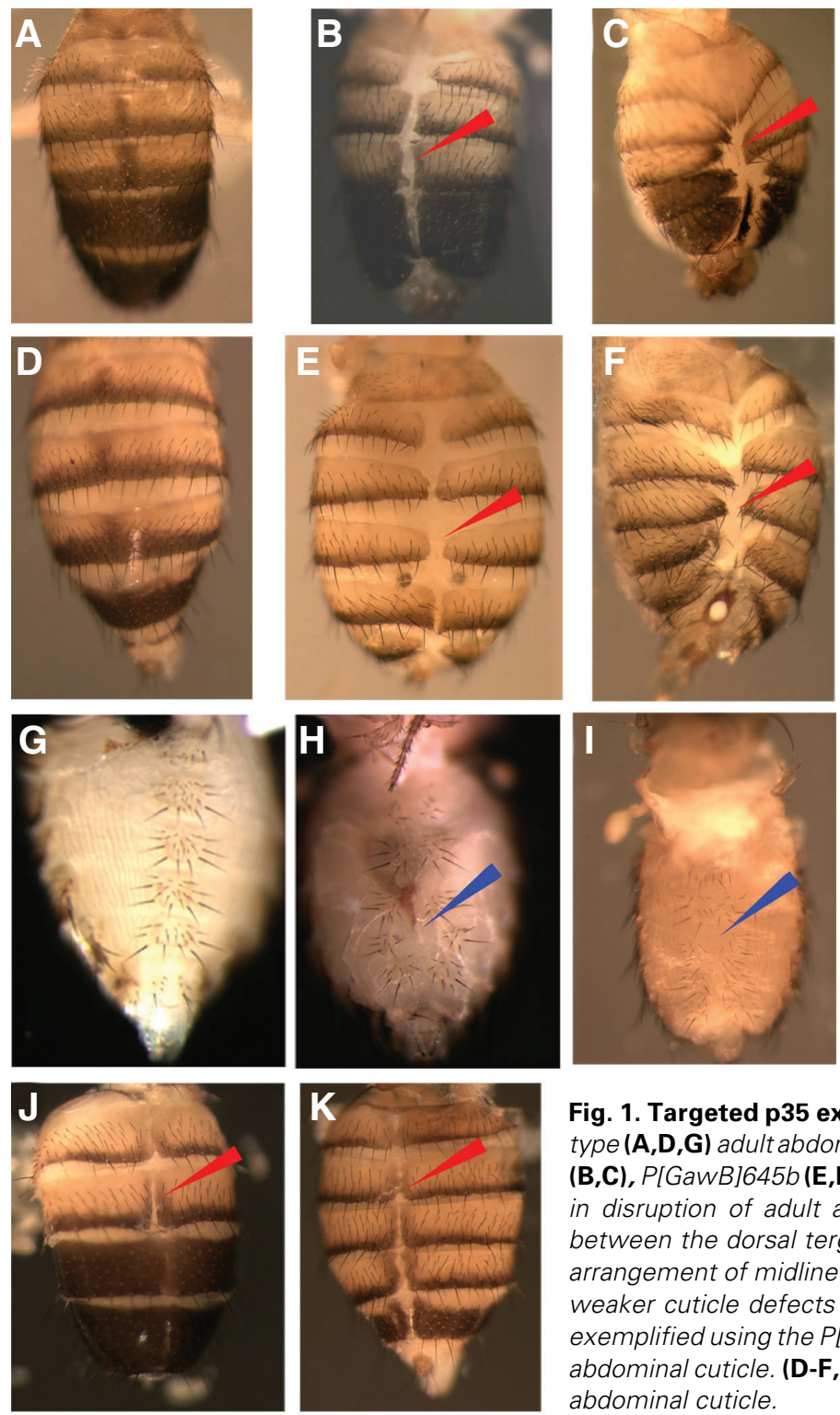

Fig. 1. Targeted p35 expression results in adult abdominal cuticle defects. Comparison of wild type $(\mathbf{A}, \mathbf{D}, \mathbf{G})$ adult abdomens with those of animals expressing $p 35(\mathbf{B}, \mathbf{C}, \mathbf{E}, \mathbf{F}, \mathbf{H}, \mathbf{I})$ via the $P[G a w B] 32 B$ (B,C), $P[G a w B] 645 b(\mathbf{E}, \mathbf{F})$, or $P[d d c-G a / 4](\mathbf{H}, \mathbf{I})$ drivers reveals that targeted caspase inhibition results in disruption of adult abdominal cuticle formation. In particular, note the strong midline splits between the dorsal tergite plates (red arrowhead in B,C,E,F) as well as disruptions in the normal arrangement of midline placed ventral sternite bristle pads (blue arrowhead in $\mathrm{H}, \mathrm{I}$ ). Similar, though weaker cuticle defects are observed for targeted expression of DIAP1 (red arrowhead in J,K) as exemplified using the P[GawB]32B (J) and PIGawB]645bb (K) drivers. (A-C,J) Dorsal aspect of male abdominal cuticle. (D-F,K) Dorsal aspect of female abdominal cuticle. (G-I) Ventral aspect of female abdominal cuticle.
$\mathrm{P}[\mathrm{GawB}] 71 \mathrm{~B}, \mathrm{P}[\mathrm{GawB}] 109-69$, and $\mathrm{P}[\mathrm{GawB}] \mathrm{c179}$ ) yielded disruptions in elaboration of the adult abdominal cuticle while 2 distinct $P[\mathrm{Gal} 4]$ lines yielded defects in the male genitalia (e.g. $\mathrm{P}[\mathrm{GawB}] 227$ ) or distal leg segments (e.g. P[GawB]DII(m23)).

For the lines that yielded abdominal cuticle defects, significant lethality was observed during pupation; p35 expression via the $\mathrm{P}[\mathrm{GawB}] 32 \mathrm{~B}$ line was nearly fully lethal. Typically, the p35expressing pupae all appeared normal during the first 24 hours APF. However, by 48 hours APF, some pupae began to exhibit arrested development and patches of necrotic tissue became apparent in the abdomen. A few pupae survived to become pharate adults with pigmented eyes, distinct bristles, and wellformed wings and legs. Some flies were incompletely emerged from the pupal cases. For most of the P[Gal4] lines, a few escaper dults successfully eclosed. All of the surviving adults and many fhe pupae exhibited stereotypic malformations in the disruptions in the joining of these cuticular plates along the and ventral midlines (Fig. $1 \mathrm{~A}-\mathrm{F}$ ). Large midline splits and grooves were present along the length of the dorsal abdomen, exposing underlying soft tissue. In addition, the lateral halves of the ventral plates often failed to join and/or exhibited bristle abnormalities along the midline (Fig. $1 \mathrm{G}-\mathrm{I}$ ). While these phenotypes were observed equally in both male and female flies, the penetrance and severity of the cuticle defects varied, suggesting that the phenotypes may be correlated with the levels of p35 expression. Consistent with this possibility, mutant phenotypes were not observed for crosses raised at $18^{\circ} \mathrm{C}$, where the levels of Gal4 expression are reduced.

The potential developmental effects of targeted expression of other cell death inhibitors, including DrosophilaDIAP1 and DIAP2, mouse $\mathrm{Bcl}-2$, and $C$. elegans $\mathrm{Ced}-9$ were also examined using relevant $P[U A S]$ lines. In contrast to the effects of $p 35$, expression of only one of these other cell death inhibitors resulted in any significant developmental phenotype. The progeny of a cross between $\mathrm{P}[\mathrm{GawB}] 32 \mathrm{~B}$ and P[UAS-DIAP1] exhibited adult defects similar to those seen for $\mathrm{p} 35$ expression, with midline splits in the abdominal cuticle. However, the severity and penetrance of this phenotype was reduced compared to that observed for p35 expression. Fewer animals exhibited midline cuticle splits and these splits often did not extend along the entire length of the abdomen (Fig. $1 \mathrm{~J}, \mathrm{~K}$ ).

Additional adult anatomical defects were also observed for targeted p35 expression and served to confirm the effectiveness of the screen in tissue-specific caspase inhibition. Thus, several P[Gal4] lines (e.g. P[GawB]227, P[drm-Gal47.1]1.1, P[GawB]32B, $P[G a w B] 645 b$, and P[ddc-Gal4.L]4.3D), yielded malformation of the external male genitalia. The male genital arches exhibited an abnormal orientation compared to wild type flies and protruded

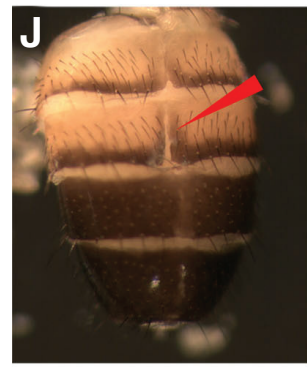



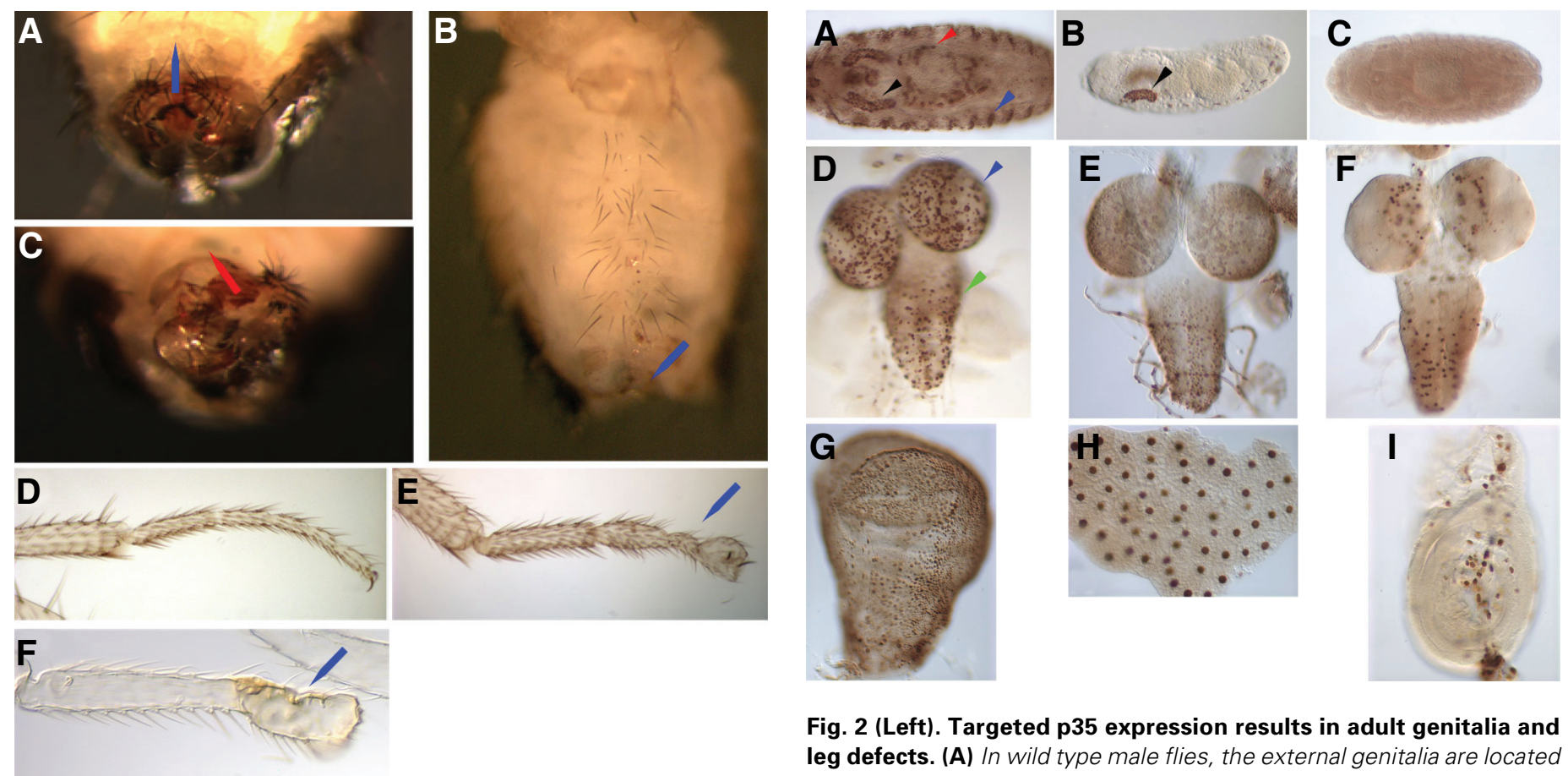

Fig. 2 (Left). Targeted p35 expression results in adult genitalia and
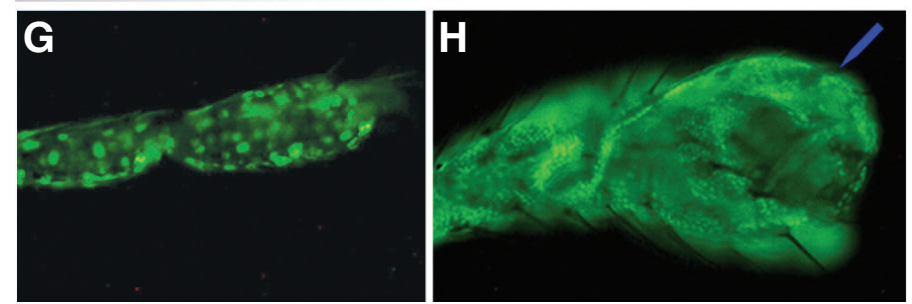

leg defects. (A) In wild type male flies, the external genitalia are located at the ventral posterior tip of the abdomen and the axis of symmetry mirrors that of the abdomen (blue arrow). (B) In male flies expressing p35 via P[GawB]227 the genitalia are misaligned and their axis of symmetry is not in line with the rest of the abdomen (red arrow). They also often exhibit a raised position compared to the wild type. (C) In a few cases, males expressing p35 exhibited a lack of external genitalia (blue arrow). Similar disruptions were observed using other P[Gal4] lines including P[GawB]32B, P[GawB]645b, P[ddc-Gal4.L]4.3D. (D,E) Distal portions of an adult leg from a P[DII-Gal4](md23) adult fly expressing GFP:NLS only $(\mathbf{D}, \mathbf{G})$, or GFP:NLS and p35 (E,F,H). Note disrupted elaboration of tarsal cuticle segments (blue arrow in E,F) and increased density of small GFP-expressing cells associated with targeted caspase inhibition (H). Legs expressing 035 often exhibited a large club-like terminal structure (blue arrow in E,F,H).

Fig. 3 (Right). P[Gal4] lines associated with adult abdominal defects exhibit distinctive expression patterns. (A) Anti- $\beta$-gal immunostaining of the progeny from crosses between P[GawB]32B, P[GawB]645b, or P[ddc-Gal4.L]4.3D and P[UAS-lacZ:NLS] strains reveal embryonic and larval expression. (A) The P[GawB]32B line drives expression in a complex pattern during embryogenesis, including the salivary gland (black arrowhead), midgut (red arrowhead), and lateral epidermal cells (blue arrowhead) in a stage 14 embryo. (B) Embryonic expression of P[GawB]645b is much more restricted and largely present in the salivary glands (black arrowhead). (C) Little or no expression is detected during embryogenesis for the PIddcGal4.LJ4.3D strain. (D,F) All three lines exhibit expression in subsets of cells in the central nervous systems of $3^{\text {rd }}$ instar larvae, including brain lobes (blue arrowhead) and ventral ganglia (green arrowhead). P[GawB]32B (D) drives the most widespread CNS expression while much of the expression for PIGawB]645b (E) is found in cells around the axon scaffold and P[ddc-Gal4.L]4.3D (F) expression is consistent with pattern of serotonergic and dopaminergic neurons. The P[GawB]645b line also exhibits strong expression in cells within the wing imaginal disc (G) and fat body (H) while $P[G a w B] 32 B$ (I) exhibits expression in a more restricted set of cells within leg imaginal discs.

aberrantly from the tip of the abdomen (Fig. 2 A,C). There was an apparent defect in normal genital rotation. In addition, a few of the progeny male flies completely lacked external genitalia (Fig. 2B). Not surprisingly, these mutant flies were all sterile. Similar findings were reported by Macías etal. (2004) for targeted expression of $\mathrm{p} 35$ by a $P$ [en-GAL4] driver. Adult anatomical defects were also observed in animals expressing p35 via the P[GawB]DII(m23) line. In particular, the distal portions of the legs were compressed and the number of tarsal segments was decreased compared to the wild type (Fig. 2 D-F). These p35-induced defects were also described by Manjon et al. (2006). We further show that consistent with a disruption in cell death, the animals expressing p35 exhibited an increased density of smaller-than-normal size cells in distal portions of the legs (Fig. $2 \mathrm{G}, \mathrm{H}$ ). These stereotypic genitalia and leg phenotypes confirm the effectiveness of the p35 expression screen.

Characterization of the P[GawB]32B and $P[$ GawB]645b lines

Three of the $P[G a 14]$ lines that exhibited the most severe abdominal phenotypes were $\mathrm{P}[\mathrm{GawB}] 32 \mathrm{~B}, \mathrm{P}[\mathrm{GawB}] 645 \mathrm{~b}$ and $P$ [ddc-Gal4.L]4.3D. The similar phenotypes resulting from p35 expression from these lines suggests that they may all share one or more common sites of expression. While P[ddc-Gal4.L]4.3D contains regulatory elements from the well characterized dopa decarboxylasegene (Konrad and Marsh 1987) the other two lines are less well defined. Thus, the corresponding genes and their patterns of expression have not been determined. To address this issue, we determined the $\mathrm{P}$ element insertion sites for $\mathrm{P}[\mathrm{GawB}] 32$ 

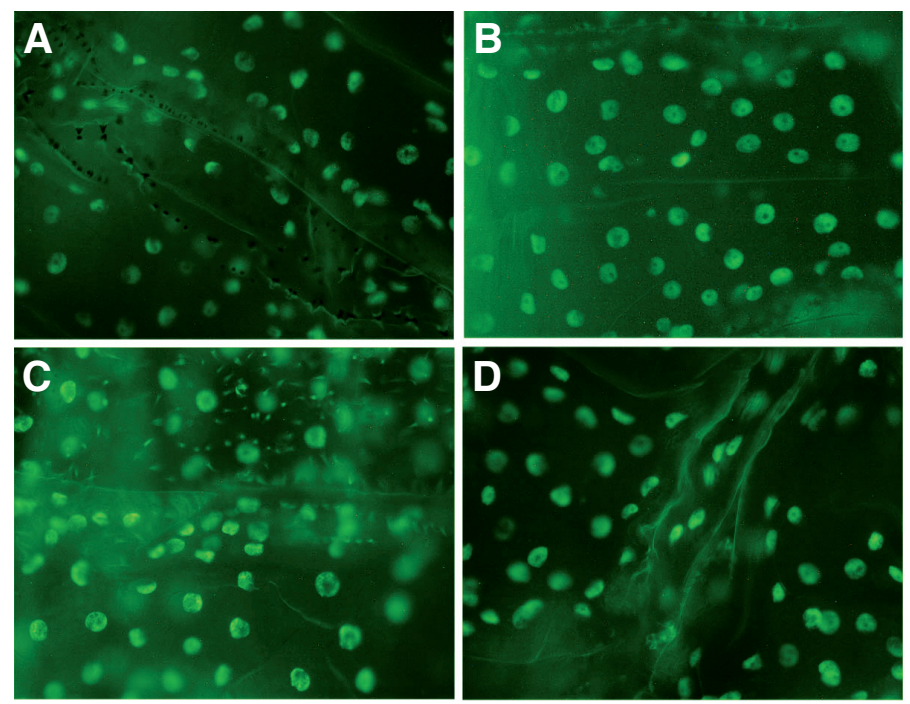

Fig. 4. $P[G a \mid 4]$ lines associated with adult abdominal defects drive expression in larval epidermal cells (LECs). Expression of GFP ::NLS via P[GawB]32B (A,B), P[GawB]645b (C), and P[ddc-Gal4.L]4.3D (D) reveals widespread labeling of $\angle E C$ nuclei along the ventral (A) and dorsal (B-D) surfaces of third instar larvae.

and $P[G a w B] 645 b P$ lines using an inverse $P C R$ approach. This analysis revealed that $P[G a w B] 32 B$ is an insertion into the largest intron of the CG13624 gene at 96A on the third chromosome (at nucleotide 20,396,601 on GB AE014297). CG13624 encodes an evolutionarily conserved basic leucine zipper transcription factor that is a member of the UPF0474 family; however, little is known about its specific functions. $P[G a w B] 645 b$ is an insertion into intron 1 of the threadgene at 72D on the third chromosome (at nucleotide 16,039,945 on GB AE014296). threadencodes DIAP1, a BIR/RING domain anti-apoptosis protein that is widely expressed (Hay et al. 1995).

The expression patterns of $\mathrm{P}[\mathrm{GawB}] 32 \mathrm{~B}$, $P[$ GawB]645b, and P[ddc-Gal4.L]4.3, were initially analyzed by crossing each line to P[UAS-LacZ]. Anti- $\beta$ galactosidase immunostaining was performed on progeny embryos and dissected larval tissues. Embryonic expression was detected for $P[G a w B] 32 B$ and $P[$ GawB $] 645 \mathrm{~b}$ in the salivary glands, epidermis, and gut (Fig. 3 A,B). P[ddc-Gal4.L]4.3D did not exhibit significant embryonic expression (Fig. 3C). In third instar larvae, expression was observed for all three lines in the central nervous system, although the precise patterns and sites of expression were distinct (Fig. 3 D-F). Both $\mathrm{P}[\mathrm{GawB}] 32 \mathrm{~B}$ and $\mathrm{P}[\mathrm{GawB}] 645 \mathrm{~b}$ drove strong expression in scattered cells throughout the larval brain lobes and the ventral ganglion. As expected, P[ddcGal4.L]4.3D exhibited expression in a pattern of cells consistent with dopamineric and serotonergic neurons within the central brain and ventral ganglion. $\mathrm{P}[\mathrm{GawB}] 32 \mathrm{~B}$ also exhibited restricted expression in the leg and wing discs while P[GawB]645b exhibited distinct expression in small numbers of cells in the leg and wing discs, as well as in the eye/antennal disc and fat bodies (Fig. 3 G-I). P[ddc-Gal4.L]4.3D exhibited no expression in the imaginal discs.

\section{Expression of P[GAL4] lines in larval epidermal cells}

In order to illuminate the basis of the adult cuticle phenotypes, it was important to determine the relevant sites of expression of the $P[G a l 4]$ lines. One possibility is that the lines might direct expression in the histoblast cells that generate the adult abdominal epidermis. However, no expression was detected in any histoblast cluster for the P[GawB]32B, P[GawB]645a, or P[ddcGal4L]4.3D lines via immunostaining of embryos or larvae (see above). We therefore examined whether the P[Gal4] lines might instead be expressed in larval epidermal cells (LECs) that are displaced by the histoblast cells during pupation. This was pursued by crossing the $P[G a w B] 32 B, P[G a w B] 645 b$ and $P[d d c-$ Gal4.L]4.3D line to a P[UAS-GFP:NLS] line and analyzing whole larval and pupal progeny. Analysis of third instar larvae revealed that each of these P[GAL4] lines exhibits prominent GFP expression in the large polyloid LECs (Fig. 4 A-D). Further analysis indicated that GFP expression is maintained in the LECs during the first 40-48 hours of the pupal stage. In general, GFP expression for each line was widespread throughout most or all of the LECs. In contrast, none of the P[Gal4] lines exhibited GFP expression in pupal histoblasts. It is striking that the $P[\mathrm{Gal} 4]$ lines all exhibited LEC expression; this finding provided compelling evidence for a potential common link between these lines and the adult cuticle defects.

\section{Targeted p35 expression disrupts replacement of larval epi- dermal cells by adult histoblasts}

We then tested the possibility that targeted p35 expression disrupts adult abdominal cuticle formation by altering LEC differentiation or survival. P[UAS-GFP]-P[UAS-p35] recombinant chromosomes were generated to simultaneously express GFP and

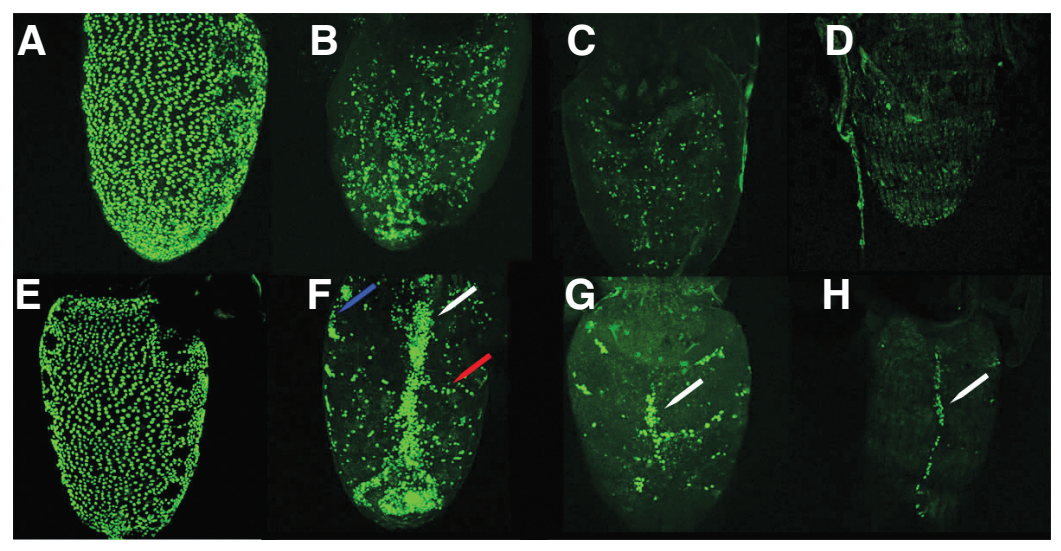

Fig. 5. P35 expression in larval epidermal cells (LECs) results in suppression of LEC loss during pupation. P[ddc-Gal4]-targeted expression of GFP::NLS alone (AD) or in combination with p35 (E-H) reveals a role for caspases in LEC fates during pupation. LECs are prominently distributed along the surface of the abdomen of control pupae at 24 hours APF (A), but by 48 hours APF (B) most of the LECs have been eliminated. By 72 hours APF (C), there are very few intact $L E C$ s remaining and by 96 hours APF (D), the LECs are completely eliminated. No difference in LEC distribution is observed in animals expressing p35 at 24 hours APF (E). However, at 48 hours APF (F) the p35-expressing pupae possess many persistent LECs that accumulate along the midline of the abdomen (white arrow), at segmental borders (red arrow), and the border between the dorsal and ventral aspect of the abdomen (blue arrow). Some of these ectopic LECs persist along the midline (white arrow) of 72 hours APF pupae (G) and 96 hours APF pharate adults $\mathbf{( H )}$, though there is a clear overall decrease in LEC numbers. 
p35. This permitted examination of LEC development in the absence of caspase activities. When driven by the $P[G a w B] 32 B$, $\mathrm{P}[\mathrm{GawB}] 645 \mathrm{~b}$ or $\mathrm{P}$ [ddc-Gal4L]4.3D lines, p35 expression did not alter the expression of GFP or behavior of the LECs during larval or early pupal stages, up to 24 hours APF (Fig. 5 A,E). However, by approximately 36 hours APF increased numbers of LECs could be detected in p35-expressing animals compared to wild type animals. These cells became tightly packed on the dorsal and ventral surface of the abdomen. LECs continued to be detected in the p35-expressing animals 48 hours APF, in contrast to wild type animals where the LECs have mostly disappeared and histoblast fusion is nearly complete (Fig. $5 \mathrm{~B}, \mathrm{~F}$ ). Unlike the wild type, these ectopic LECs were still detected at 72 hours APF (Fig. 5 C,G) and accumulated mostly along the midline on both the dorsal and ventral sides of the abdomen as well as along the segmental boundaries. These sites correspond to the cuticle splits observed in the adult abdomen (see Fig. 1). Some p35-expressing LECs persisted along the abdominal midline up to the pharate adult stage, well after all LECs are normally eliminated (Fig. $5 \mathrm{D}, \mathrm{H}$ ). Interestingly, despite the p35-mediated persistence of some LECs throughout pupation, there still was a significant decrease in their overall numbers. This suggests that many p35-expressing LECs were ultimately eliminated.

\section{Cell death in the pupal abdomen}

The normal loss of LECs during pupation can be inhibited by p35, suggesting that this loss is the result of a caspase-dependent programmed cell death process. To directly visualize dying LECs during pupation, we stained whole mount abdominal tissue with ethidium homodimer-1 (EthD-1), a membrane impermeant high affinity nucleic acid binding dye that serves as a cell viability indicator (Weidman et al. 2008). In wild type pupae 13 hour APF, EthD-1 staining was able to detect dying cells in the imaginal discs (Fig. 6 A,B). When used to examine wild type and p35-expressing pupae at 36 hours APF, the wild type animals, exhibited scattered EthD-1 labeled cells along the margins of contact between the histoblasts and LECs (Fig. 6 C,D). In contrast, few EthD-1 labeled cells were detected in the animals expressing p35 (Fig. 6 E,F). This suggests that the LECs are normally eliminated during pupation by programmed developmental cell death that occurs as the histoblast cells have proliferated and are migrating across the abdomen. Much of this death is delayed or blocked by p35 expression in the LECs. As p35 does not block the activity of the initiator caspase Dronc (Hawkins et al. 2000), we also examined the effects of expressing a dominant/negative form of Dronc via P[UAS- Dronc ${ }^{\mathrm{C} 318 \mathrm{~S}}$ ] (Huh et al. 2004) using the P[GawB]32B and $P$ [ddc-Gal4] lines. No lethality or cuticle defects were observed in any of the Dronc ${ }^{\mathrm{C} 318 \mathrm{~S}}$-expressing progeny (data not shown).

\section{Discussion}

\section{Programmed cell death and development in Drosophila}

The baculovirus p35 protein is a potent inhibitor of many caspases and can effectively block a wide array of developmental and pathological cell deaths in Drosophila (Davidson and Steller 1998; Hay et al. 1994). In this study, we utilized the Gal4/UAS system to target p35 expression to many different tissues and cell types in developing Drosophila. For the most part, p35 expression did not result in organismal lethality or significant anatomical
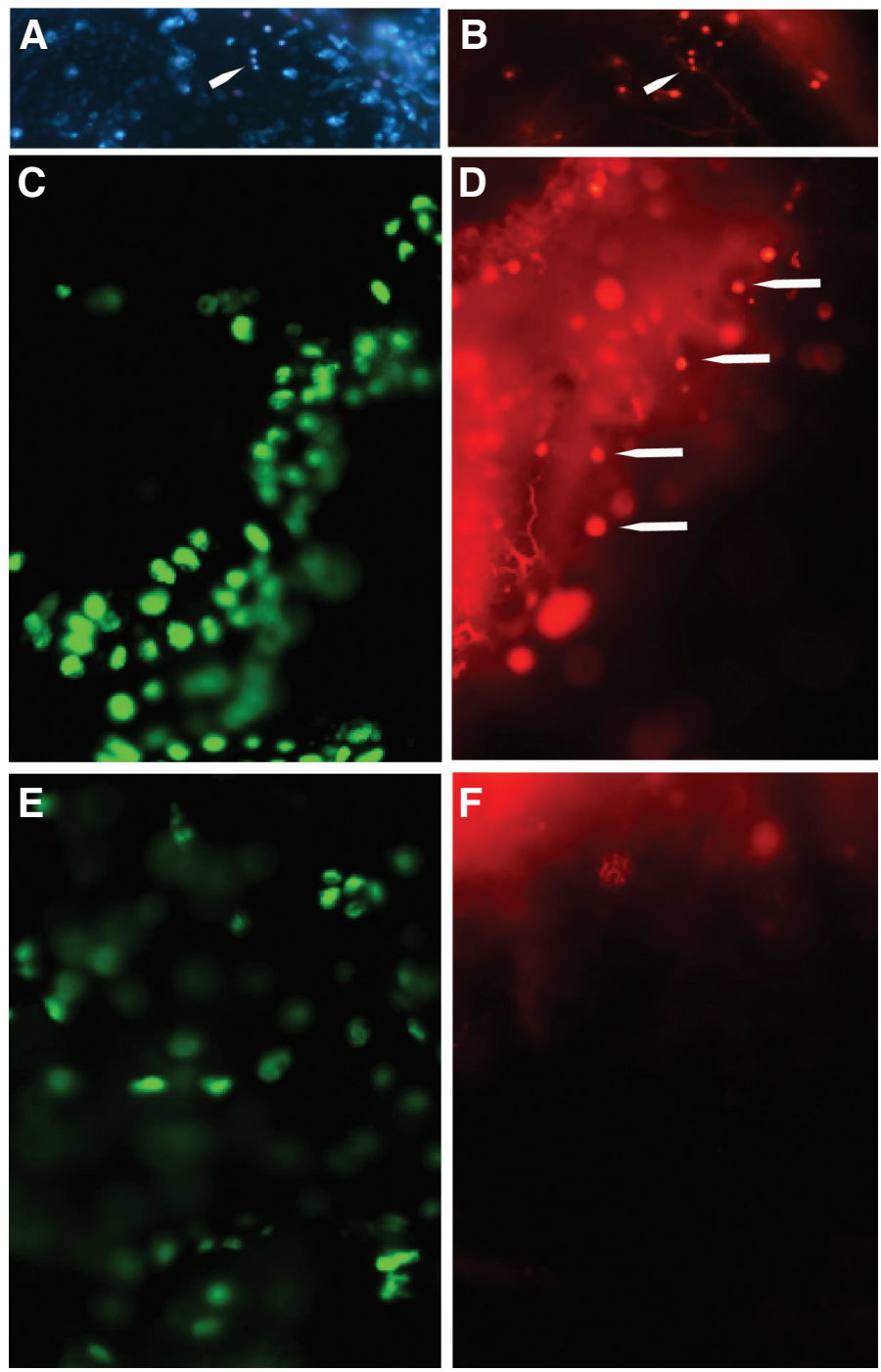

Fig. 6. Larval epidermal cells (LECs) undergo cell death during pupation. (A) DAPI staining reveals nuclei in a leg imaginal disc of a pupae at 13 hour APF (note nuclei indicated by white arrow). (B) In this same disc the presence of dying cells (red) is revealed via nuclear accumulation of Ethidium-1 homodimer (note nuclei indicated by white arrow). (C) In P[GawB]32B, P[UAS-GFP] animals 36 hours APF, GFP expression is detected in scattered LECs. (D) Ethidium-1 homodimer staining (red) of a 36 hour APF P[GawB]32B, P[UAS-GFP] animal reveals widespread labeling of dying cells (white arrows). (E) In P[GawB]32B, PIUAS-p35]-P[UAS-GFP] animals 36 hour APF, GFP expression is also detected in scattered LECs. However, in these animals ethidium-1 homodimer fails to detect dying cells (F).

defects. While the screen was limited in scope, the results nonetheless suggest that many tissues are able to complete organogenesis in the absence of, or greatly decreased cell death. This is consistent with significant plasticity in the ability of developing Drosophila tissues to accommodate changes in cell numbers and survival patterns (reviewed in Martin et al. 2009; Fan and Bergmann 2008)

During normal development, extensive cell death is associated with cell differentiation and organogenesis during embryogenesis and pupation, stages when the two very different Drosophila life 
forms are generated. In this screen, the anatomical defects observed all affected the elaboration of adult rather than larval structures. In addition, the lethality detected occurred during pupation, not embryogenesis. Several potential factors may underlie this apparent bias. First, the adult is more anatomically complex than the larva. Adult flies possess a number of sophisticated appendages and specialized structures whose proper elaboration requires the coordinated behavior of greater numbers of cells and cell types. This may require more finely tuned patterns of cell death for successful completion. Furthermore, during pupation, it is necessary to eliminate entire larval organs and tissues to permit construction of adult structures. This involves the removal of an entire scaffold of larval structures. In contrast, much of the cell death that occurs during embryogenesis is involved in establishing proper sizes of cell populations and removing specific and restricted cells with transient functions. Finally, there may be greater inherent flexibility in the formation of more generalized larval features. Analysis of embryos carrying different doses of the bicoidgene have indicated that large variations in the initial patterning of the blastoderm can ultimately be accommodated to generate normally proportioned larvae (Namba et al. 1997). The role of cell death in formation of the Drosophila larva may be more comparable to its role in development of the adult $C$. elegans roundworm than to pupation and generation of the adult fly. Overall, our findings indicate that a blockade of caspase activities can result in significant and highly specific anatomical defects.

\section{Caspase functions are essential for development of the male genitalia and leg tissue}

Several P[Gal4] lines were associated with p35-mediated disruptions in the adult male genitalia. In particular, the external genital structures were atypically positioned at the tip of the abdomen and failed to properly rotate. Very similar phenotypes have been described for some alleles of the Wrinkledand Pvf1 genes (Macías et al. 2004; Abbott and Lengyel 1991). Wrinkled encodes HID, a member of the RHG family of apoptosis activators (Grether et al., 1995) and Pvf1 encodes a Drosophila Platelet Derived Growth Factor homolog (Macías et al. 2004). These proteins likely regulate a caspase cascade required for male genital morphogenesis. Interestingly, not all the RHG genes are required for this process. Thus, reaper mutants do not exhibit disruptions in external male genitalia, though the mutant males are sterile (Peterson et al. 2002). The disruptions in leg development associated with P[DII-Gal4]-directed p35 expression confirm that caspase activities are important for normal leg morphogenesis (Manjón et al. 2006). In the absence of caspase activities, we observed defects in elaboration of tarsal leg segments associated with increased cellular densities and smaller cell sizes. It is unclear if the ectopic cells correspond solely to those rescued from death, or if they might include cells generated via compensatory proliferation processes that have been observed in other imaginal tissues (reviewed in Martin et al. 2009; Fan and Bergmann 2008). Interestingly, similar leg or abdomen phenotypes were not observed for widespread/ubiquitous expression of $p 35$. This suggests that $\mathrm{P}[\mathrm{Gal} 4]$ lines can provide spatial and temporal patterns of expression that uniquely reveal the importance of cell death in specific morphogenetic processes.

\section{A model for p35-induced adult cuticle defects}

In this study we describe a novel developmental role for caspase-mediated programmed cell death in abdominal morphogenesis. In particular, caspase-dependent elimination of LECs is critical for proper migration of histoblast cells and formation of the adult abdominal cuticle. Previous studies have shown that when histoblast cells are ablated via irradiation during larval stages, the LECs survive and ultimately secrete adult cuticle during pupation (Madhavan and Madhavan 1984). In addition, the proliferation and spreading of histoblasts and elimination of LECs involves intimate communication between these cell populations, mediated in part by secretion of Dpp from LECs (Ninov et al. 2010; 2007). The LECs are ultimately extruded from the developing abdominal epithelium and phagocytosed by circulating hemocytes. From these and our data, a model emerges for the role of caspases in normal adult abdominal cuticle formation (Fig. 7). Specifically, the LECs appear to undergo a histoblast-induced, caspase-dependent programmed cell death during pupation. Death and removal of the LECs is essential for the differentiating histoblast cells to fully migrate and cover the adult abdomen. Targeted p35 expression can block or delay LEC death and the persistent LECs are pushed to the margins along the dorsal and

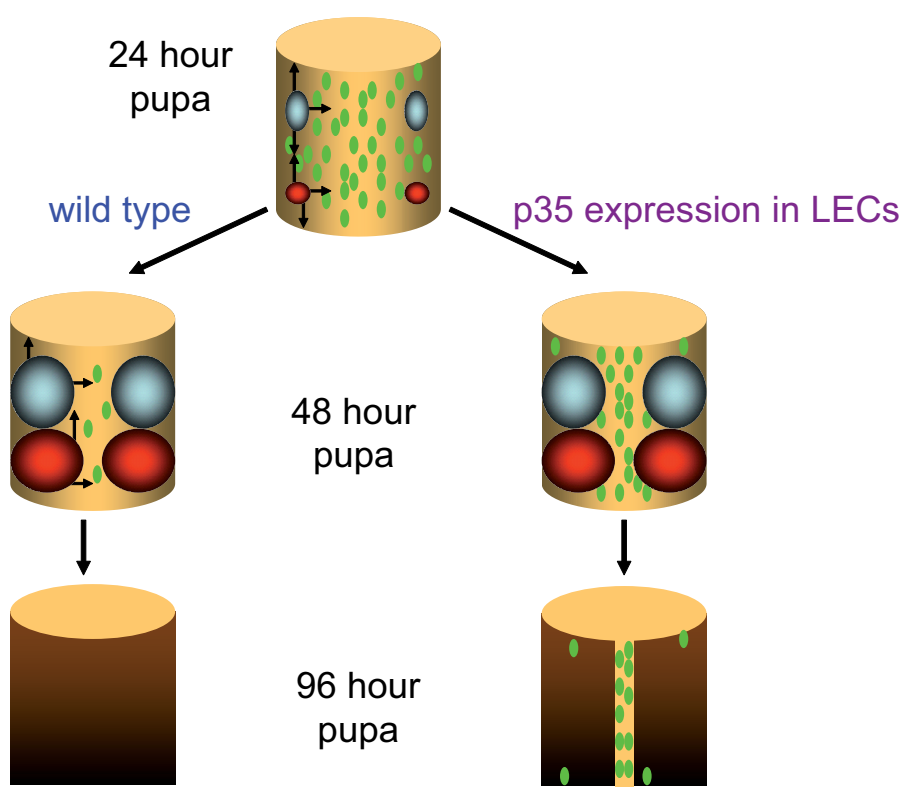

Fig. 7. Model for the role of programmed cell death in adult abdomen morphogenesis. During early pupation (24 hour pupa), larval epidermal cells (LECs, small green ovals) are distributed along the surface of the abdominal epidermis. Cells of the histoblast nests, including the anterior dorsal nest (ADN; gray ovals) and posterior dorsal nest (PDN; red ovals) proliferate and migrate outwards (arrows). By 48 hours APF, most of the LECs have been eliminated in wild type animals and the ADN and PDN cells have covered much of the abdominal surface. In contrast, many LECs are still detected in animals expressing p35 in the LECs. By 96 hours APF (pharate adult), the LECs are absent in wild type animals and the ADN and PDN cells have merged and secrete the adult dorsal abdominal cuticle plates (dark brown). In animals expressing p35, the ADN and PDN cells do not properly merge and some LECs continue persist along the dorsal midline and segmental boundaries. These defects result in large splits along the midline of the adult cuticle. 
ventral midlines. The absence of histoblast derivatives in these locations disrupts normal cuticle formation and leads to characteristic splits in adult the adult abdomen. These cuticle splits suggest that rescued LECs do not efficiently contribute to the adult cuticle. This observation contrasts with the ability of LECs to secrete adult cuticle in the absence of histoblasts (Madhavan and Madhavan 1984) and suggests that p35 expression may also disrupt caspase-dependent cuticle synthesis by the LECs. Interestingly, p35 did not block the death of all LECs as significantly fewer LECs remained at end of pupation than were present at its beginning. This suggests that $\mathrm{p} 35$ expression may act largely to delay LEC deaths, and that specific subsets of LECs may have distinct sensitivities to p35.

The p35-induced disruption of histoblasts and LECS that resulted in the midline cuticle splits in the abdomen is reminiscent of mammalian cleft palate phenotypes (Gritli-Linde 2007). Cleft palate formation may involve defects in the elimination of the midline epithelial seam that is generated during fusion of midline edge epithelial cells (reviewed in Nawshad 2008). In the absence of this cell elimination, palatal shelf fusion is not completed and a midline cleft results. In both these developmental processes, disruptions in normal cell death patterns may contribute to the accumulation of ectopic cells between sheets of migrating lateral cells, resulting in distinctive midline anatomical defects. Thus, programmed cell death may be a conserved mechanism to facilitate fusion of migrating populations of cells that must join together to generate a seamless tissue structure.

\section{Materials and Methods}

\section{Drosophila strains}

The P[Gal4] strains listed in Table 1 were all obtained from the Bloomington Bloomington Drosophila Stock Center (http:// flystocks.bio.indiana.edu), as were the P[UAS-p35.H]BH1 and P[UASp35.H]BH2 strains. P[GawB]52a, P[UAS-DIAP1] and P[UAS-DIAP2] were previously described (Zhou et al. 1997; Wing et al. 2001). The P[UASP35,UAS-LacZ::GFP]/TM3 and P[UAS-P35, UAS-LacZ:GFP]/CyO strains were generated via meiotic recombination. The P[UAS-dnDronc $\left.{ }^{\left[{ }^{[318 S}\right]}\right]$, P[UAS-ced-9], and P[UAS-bcl2] were kindly provided by B. Hay, H. Miura, and $\mathrm{B}$. Mignotte respectively. All stocks and crosses were raised at $25^{\circ} \mathrm{C}$ unless otherwise noted.

\section{Inverse PCR}

Genomic DNA was isolated from the P[GawB]32B and $\mathrm{P}[\mathrm{GawB}] 645 \mathrm{~b}$ strains essentially as described in Wing et al. (2002). Aliquots of the genomic DNA were digested with Sau3A restriction enzyme and then heated at $85^{\circ} \mathrm{C}$ for 15 minutes. The digested DNA was incubated with T4 DNA ligase overnight at $18^{\circ} \mathrm{C}$. The ligated DNA was precipitated with $\mathrm{EtOH}$, resuspended, and used in PCR assays.

The primers used for $\mathrm{PCR}$ analysis of $\mathrm{P}[\mathrm{GawB}] 645 \mathrm{~b}$ and $\mathrm{P}[\mathrm{GawB}] 32 \mathrm{~B}$ genomic DNA were:

3' Primer: 5'-CGCTGTCTCACTCAGACTCAATAC-3' and 5' Primer:

\section{5'-AACCCTTAGCATGTCCGTGG-3'.}

These primers are complementary to the 3 ' and 5' $P$ element ends. The PCR reactions were set up as follows:

$20 \mu \mathrm{L}$ of purified ligated genomic DNA, $2 \mu \mathrm{L}$ of the 5' and 3' primers, $2 \mu \mathrm{L}$ of Taq polymerase and $25 \mu \mathrm{L}$ of $\mathrm{ddH}_{2} \mathrm{O}$ were added to the Ready-Mix tube (Sigma Aldrich).

The PCR products were purified from agarose gels using the QIAquick Gel Extraction Kit (Qiagen). Aliquots of the purified DNA were sent to Davis Sequencing (Davis, CA) for DNA sequence analysis. The se- quences obtained were used to identify corresponding regions of Drosophila Melanogastergenomic DNA via Blastn searches using the BLAST utility at Flybase (www.flybase.org/).

\section{Immunocytochemisty}

Anti- $\beta$-galactosidase immunostaining was performed on whole embryos and dissected $3^{\text {rd }}$ instar larval tissues from crosses between P[GawB]32B, P[GawB]645b and P[7ddc-Gal4.L]4.3D P[UAS-LacZ::GFP] strains essentially as described in Zhou et al. (1997) or Mukherjee et al. (2000) using an anti- $\beta$-gal MAb (Promega) and biotinylated anti-mouse IgG secondary antibody (Vector Laboratories). Antibody binding was visualized via $D A B / H R P$ histochemistry using the Elite $A B C$ detection kit (Vector Laboratories). The stained embryos were dehydrated in an $\mathrm{EtOH}$ series, cleared in methyl salicylate, and mounted Permount mounting medium (Fisher Scientific). The stained tissues were analyzed and documented via DIC optics.

\section{Confocal microscopy}

Staged larvae and pupae from the P[Gal4] and P[UAS-LacZ::GFP] crosses were collected, rinsed, and the pupal case removed. The animals were mounted in Vectashield with DAPI (Vector Laboratories, \# H-1200) and GFP was visualized via confocal microscopy using a Zeiss LSM 510 Meta Confocal Microscope at the University of Massachusetts Amherst Central Microscope Facility (http://www.bio.umass.edu/microscopy/).

\section{DAPI and ethidium homodimer-1 staining}

Staged pupae were collected and the pupal cases were cleaned in $1 \times P B S$ and then incubated at $50 \infty \mathrm{CddH}_{2} \mathrm{O}$ for 5-10 minutes. The pupae were transferred to a glass slide with drop of PBS and bissected either dorso-ventrally or laterally with a razor blade. The dorsal half was then transferred to a glass bottom dish with PBS. Unwanted internal organ debris was removed with forceps and by gently squirting PBS several times at the inner surface of the integument. After internal organs were removed, the epithelial layer of the cuticle was removed from the pupal case.

For DAPI staining, the pupal cuticle was mounted in Vectashield with DAPI (Vector Labs) and visualized via epifluorescence. For Ethidium-1 homodimer staining prepared pupal tissue was incubated for 10 minutes at room temperature with $10 \mathrm{uM}$ ethidium homodimer-1 (Invitrogen). Tissue was washed in 1xPBS and mounted in Vectashield and visualized via fluorescence microscopy.

\section{Acknowledgements}

The authors thank Bruce Hay, H. Miura, B. Mignotte and the Bloomington Drosophila Stock Center for providing various fly strains. We are grateful for the technical assistance provided by Nadire Ramadan, Tia Coleman, Barbara Schreader, and Kenneth Vasques. This work was supported in part by NIH grant AG025866 to J.R.N. and a UMASS Commonwealth College Research Fellowship to R.S.K.

\section{References}

ABBOTT, M.K., LENGYEL, J.A. (1991). Embryonic head involution and rotation of male terminalia require the Drosophila locus head involution defective. Genetics 129: 783-789.

BAEHRECKE, E. (2002). How death shapes life during development. Nat Rev Mol Cell Bio/3: 779-787.

CHEW, S.K., AKDEMIR, F., CHEN, P., LU, W.-J., MILLS, K., DAISH, T., KUMAR, S., RODRIGUEZ, A., ABRAMS, J.M. (2004). The apical caspase dronc governs programmed and unprogrammed cell death in Drosophila. Dev Cel/7: 897-907.

CHOWDHURY, I., THARAKAN, B., BHAT, G.K. (2008). Caspases - an update. Comp Biochem Physiol B Biochem Mol Biol151: 10-27.

CHRISTOFFERSON, D.E., YUAN, J. (2009). Necroptosis as an alternative form of programmed cell death. Curr Opin Cell Bio/22: 263-268. 
CLEM, R.J. (2007). Baculoviruses and apoptosis: a diversity of genes and responses. Curr Drug Targets 8: 1069-1074

DAISH, T.J., MILLS, K., KUMAR, S. (2004). Drosophila caspase DRONC is required for specific developmental cell death pathways and stress-induced apoptosis. Dev Cel/7: 909-915.

DANON, A., ROTARI, V.I., GORDON, A., MAILHAC, N., GALLOIS, P. (2004). Ultraviolet-C overexposure induces programmed cell death in Arabidopsis, which is mediated by caspase-like activities and which can be suppressed by caspase inhibitors, p35 and Defender against Apoptotic Death. J Biol Chem 279: 779-787.

DAVIDSON, F.F., STELLER, H. (1998). Blocking apoptosis prevents blindness in Drosophila retinal degeneration mutants. Nature 391: 587-591.

DELA CRUZ, W.P., FRIESEN, P.D., FISHER, A.J. (2001). Crystal structure of baculovirus $\mathrm{p} 35$ reveals a novel conformational change in the ractive site loop after caspase cleavage. J Biol Chem 276: 32933-32939.

DOMINGOS, P.M., STELLER, H. (2007). Pathways regulating apoptosis during patterning and development. Curr Opin Genet Dev 17: 294-299.

ELLIS, H., HORVITZ, H. (1986). Genetic control of programmed cell death in the nematode C. elegans. Cel/44: 817-829.

FAN, Y., BERGMANN, A. (2008). Apoptosis-induced compensatory proliferation. The cell is dead. Long live the cell! Trends Cell Bio/18: 467-473.

FEINSTEIN-ROTKOPF, Y., ARAMA, E. (2009). Can't live without them, can live with them: roles of caspases during vital cellular processes. Apoptosis 14: 980 995.

FRISTROM, D., FRISTROM, J.W. (1993). The metamorphic development of the adult epidermis. In: The Development of Drosophila melanogster(Eds. M. Bate, A. Martinez-Arias), Cold Spring Harbor NY:Cold Spring Harbor Laboratory Press, pp 843-897

GALLUZZI, L., JOZA, N., TASDEMIR, E., MAIURI, M.C., HENGARTNER, M., ABRAMS, J.M., TAVERNARAKIS, N., PENNINGER, J., MADEO, F., KROEMER, G. (2008). No death without life: vital functions of apoptotic effectors. Cel/ Death Differ 15: 1113-1123.

GRETHER, M., ABRAMS, J.M., AGAPITE, J., WHITE, K., STELLER, H. (1995). The head involution defective gene of Drosophila Melanogaster functions in programmed cell death. Genes Dev 15: 1694-1708.

GRITLI-LINDE, A. (2007). Molecular control of secondary palate development. Dev Bio/301: 309-326.

HAWKINS, C.J., YOO, S.J., PETERSON, E.P., WANG, S.L., VERNOOY, S.Y., HAY, B.A. (2000). The Drosophilacaspase DRONC cleaves following glutamate or aspartate and is regulated by DIAP1, HID, and GRIM. J Biol Chem 275: 27084-27093.

HAY, B.A., GUO, M. (2006). Caspase-dependent cell death in Drosophila. Annu Rev Cell Dev Biol22: 623-650.

HAY, B., WASSARMAN, D., RUBIN, G. (1995). Drosophilahomologs of baculovirus inhibitor of apoptosis proteins functions to block cell death. Cel/7: 1253-1262.

HAY, B.A., WOLFF, T., RUBIN, G.M. (1994). Expression of baculovirus P35 prevents cell death in Drosophila. Development 120: 2121-2129.

HOTCHKISS, R.S., STRASSER, A., MCDUNN, J.E., SWANSON, P.E. (2009). Cell death. N Eng/J Med 361: 1570-1583.

HUH, J.R., GUO, M., HAY, B.A. (2004). Compensatory proliferation induced by cell death in the Drosophila wing requires activity of the apical cell death caspase dronc in a nonapoptotic role. Curr Biol14: 1262-1266.

IZQUIERDO, M., GRANDIEN, A., CRIADO, L.M., ROBLES, S., LEONARDO, E., ALBAR, J.P., DE BUITRAGO, G.G., MARTÍNEZ-A, C. 1999. Blocked negative selection of developing $T$ cells in mice expressing the baculovirus p35 caspase inhibitor. EMBO J18: 156-66.

KONRAD, K.D., MARSH, J.L. (1987). Developmental expression and spatia distribution of dopadecarboxylase in Drosophila Dev Bio/122: 172-185.

KRYSKO, D.V., VANDEN BERGHE, T., PARTHOENS, E., D'HERDE, K., VANDENABEELE, P. (2008). Methods for distinguishing apoptotic from necrotic cells and measuring their clearance. Methods Enzymo/442: 307-341.

LETTRE, G., HENGARTNER, M.O. (2006). Developmental apoptosis in C. elegans: a complex CEDnario. Nat Rev Mol Cell Biol7: 97-108.

LI, J., YUAN, J. (2008). Caspases in apoptosis and beyond. Oncogene 27: 61946206.
LAMKANFI, M., FESTJENS, N., DECLERCQ, W., VANDEN BERGHE, T., VANDENABELE, P. (2007). Caspases in cell survival, proliferation, and differentiation. Cell Death Diff 14: 44-55.

MACE, P.D., SHIRLEY, S., DAY, C.L. (2010). Assembling the building blocks: structure and function of inhibitor of apoptosis proteins. Cel/ Death Diff 17: 4653.

MACÍAS, A., ROMERO, N.M., MARTÍN, F., SUÁREZ, L., ROSA, A.L., MORATA, G. (2004). PVF1/PVR signaling and apoptosis promotes the rotation and dorsal closure of the Drosophila male terminalia. Int J Dev Bio/48: 1087-1094.

MADHAVAN, M., MADHAVAN, K. (1984). Do larval epidermal cells possess the blueprint for adult pattern in Drosophila? J Embryol Exp Morph 82: 1-8.

MADHAVAN, M., MADHAVAN, K. (1980). Morphogenesis of the epidermis of adult abdomen of Drosophila. J Embryol Exp Morph 60: 1-31.

MADHAVAN, M., SCHNEIDERMAN, H. (1977). Histological analysis of the dynamics of growth of the imaginal discs and histoblast nests during the larval development of Drosophila Melanogaster. Wilhelm Roux Arch Dev/ Biol 183: 269-305.

MANJON, C., SANCHEZ-HERRERO, E., SUZANNE, M. (2006). Sharp boundaries of Dpp signaling trigger local cell death required for Drosophilaleg morphogenesis. Nat Cel/ Bio/9: 57-63.

MARTIN, F.A., PÉREZ-GARIJO, A., MORATA, G. (2009). Apoptosis in Drosophila compensatory proliferation and undead cells. Int J Dev Bio 53: 1341-1347.

MEIER, P., FINCH, A., EVAN, G. (2000). Apoptosis in development. Nature 407: 796-801.

MUKHERJEE, A., SHAN, X., MUTSUDDI, M., MA, Y., NAMBU, J.R. (2000). The Drosophila sox gene, fish-hook, is required for postembryonic development. Dev Biol217: 91-106.

NAMBA, R., PAZDERA, T.M., CERRONE, R.L., MINDEN, J.S. (1997). Drosophila embryonic pattern repair: how embryos respond to bicoid dosage alteration. Development 124: 1393-1403.

NAWSHAD, A. (2008). Palatal seam disintegration: To die or not to die? That is no longer the question. Dev Dyn 237: 2643-2656.

NINOV, N., MENEZES-CABRAL, S., PRAT-ROJO, C., MAJÓN C., WEISS, A., PYROWOLAKIS G., AFFOLTER, M., MARTÍN-BLANCO, E. (2010). Dpp signaling directs cell motility and invasiveness during epithelial morphogenesis. Curr Bio/20: 513-520.

NINOV, N., CHIARELLI, D.A., MARTÍN-BLANCO, E. (2007). Extrinsic and intrinsic mechanisms directing epithelial cell sheet replacement during Drosophila metamorphosis. Development 134: 367-379.

ORME, M., MEIER, P. (2009). Inhibitor of apoptosis proteins in Drosophila. gatekeepers of death. Apoptosis 14: 950-960

PETERSON, C., CARNEY, G.E., TAYLOR, B.J., WHITE, K. (2002). Reaper is required for neuroblast apoptosis during Drosophila development. Development 129: 1467-1476.

RIEDL, S.J., SHI, Y. (2004). Molecular mechanisms of caspase regulation during apoptosis. Nat Rev Mol Cell Bio/5: 897-907.

SALVESEN, G.S., RIEDL, S.J. (2008). Caspase mechanisms. Adv Exp Med Biol 615: 13-23.

STELLER, H. (2008). Regulation of apoptosis in Drosophila. Cell Death Differ 15: 1132-1138.

SUGIMOTO, A., FRIESEN, P.D., ROTHMAN, J.H. (1994). Baculovirus p35 prevents developmentally programmed cell death and rescues a ced-9 mutant in the nematode Caenorhabditis elegans. EMBO J13: 2023-2028.

TAYLOR, R.C., CULLEN, S.P., MARTIN, S.J. (2008). Apoptosis: controlled demolition at the cellular level. Nat Rev Mol Cell Bio/9: 231-241.

WANG, J., LENARDO, M.J. (2000). Roles of caspases in apoptosis, development, and cytokine maturation revealed by homozygous gene deficiencies. J Cel/ Sci 113: $753: 757$.

WEIDMAN, D., SHAW, J., BEDNARCZYK, J., REGULA, K.M., YURKOVA, N., ZHANG, T., AGUILAR, F., KIRSHENBAUM, L.A. (2008). Dissecting apoptosis and intrinsic death pathways in the heart. Methods Enzymo/4466: 277-285.

WING, J.P., SCHWARTZ, L.M., NAMBU, J.R. (2001). The RHG motifs of Drosophila Reaper and Grim are important for their distinct cell death-inducing abilities. Mech Dev102: 193-203.

WING, J.P., KARRES, J.S., OGDAHL, J.L., ZHOU, L., SCHWARTZ, L.M., NAMBU, 
J.R. (2002). Drosophila sickle is a novel grim-reaper cell death activator. Curr Biol 12: 131-135.

XU, D., LI, Y., ARCARO, M., LACKEY, M., BERGMANN, A. (2005). The CARDcarrying caspase Dronc is essential for most, but not all, developmental cell death in Drosophila. Development 132: 2125-2134.

Xu, G., RICH, R.L., STEEGBORN, C., MIN, T., HUANG, Y., MYSZKA, D.G., WU, $H$. (2003). Mutational analyses of the p35-caspase interaction. A bowstring kinetical model of caspase inhibition by p35. J Biol Chem 278: 5455-5461.

XU, G., CIRILLI, M., HUANG, Y., RICH, R.L., MYSZKA, D.G., WU, H. (2001). Covalent inhibition revealed by the crystal structure of the caspase-8/p35 complex. Nature 410: 494-497.

XUE, D., HORVITZ, H.R. (1995). Inhibition of the Caenorhabditis elegans cell- death protease CED-3 by a CED-3 cleavage site in baculovirus p35 protein Nature 377: 248-251.

YUAN, J., SHAHAM, S., LEDOUX, S., ELLIS, H.M., HORVITZ, H.R. (1993). The C elegans cell death gene ced-3 encodes a protein similar to mammalian interleukin1 beta-converting enzyme. Cel/75: 641-652.

ZHENG, T.S., HUNOT, S., KUIDA, K., FLAVELL, R.A. (1999). Caspase knockouts: matters of life and death. Cell Death Differ6: 1043-1053.

ZHOU, L., SCHNITZLER, A., AGAPITE, J., SCHWARTZ, L.M., STELLER, H., NAMBU, J.R. (1997). Cooperative functions of the reaper and head involution defective genes in the programmed cell death of Drosophila central nervous system midline cells. Proc Natl Acad Sci USA 94: 5131-5136.

\section{Further Related Reading, published previously in the Int. J. Dev. Biol.}

See our recent Special Issue Placenta edited by Joan S. Hunt and Kent L. Thornburg at: http://www.ijdb.ehu.es/web/contents.php?vol=54\&issue=2-3

Both jnk and apoptosis pathways regulate growth and terminalia rotation during Drosophila genital disc development Sergio Benitez, Claudia Sosa, Nicolás Tomasini and Ana Macías Int. J. Dev. Biol. (2010) 54: 643-653

Apoptosis in Drosophila: compensatory proliferation and undead cells Francisco A. Martín, Ainhoa Peréz-Garijo and Ginés Morata Int. J. Dev. Biol. (2009) 53: 1341-1347

Metamorphosis of Hydractinia echinata (Cnidaria) is caspase-dependent Stefanie Seipp, Karola Wittig, Beate Stiening, Angelika Böttger and Thomas Leitz Int. J. Dev. Biol. (2006) 50: 63-70

A generalized caspase inhibitor disrupts early mammalian development Zahra Zakeri Richard A. Lockshin, Luis-Miguel Criado-Rodríguez and Carlos Martínez-A Int. J. Dev. Biol. (2005) 49: 43-51

\section{Genetic control of epidermis differentiation in Drosophila}

François Payre

Int. J. Dev. Biol. (2004) 48: 207-215

Programmed cell death is not a necessary prerequisite for fusion of the fetal mouse palate Sachiko Takahara, Toshiya Takigawa and Kohei Shiota Int. J. Dev. Biol. (2004) 48: 39-46

5 yr ISI Impact Factor $(2008)=3.271$

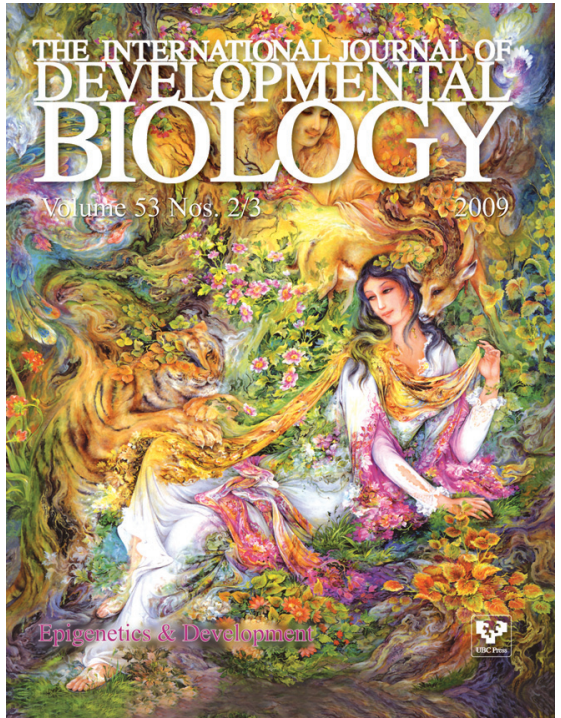

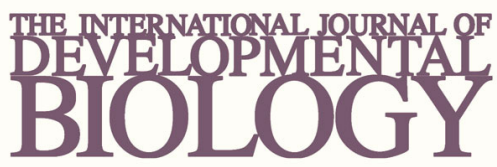

Volume 54 Nos. $6 / 7$
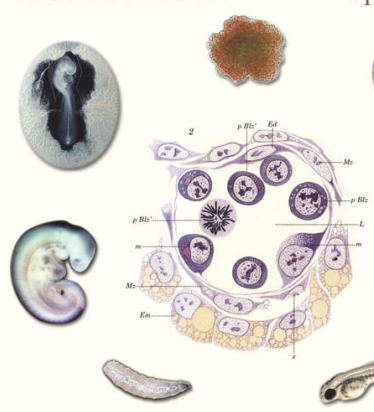

Developmental Hematopoiesis
Special Issue
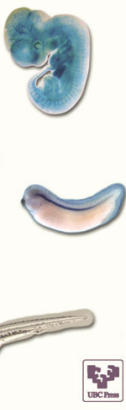

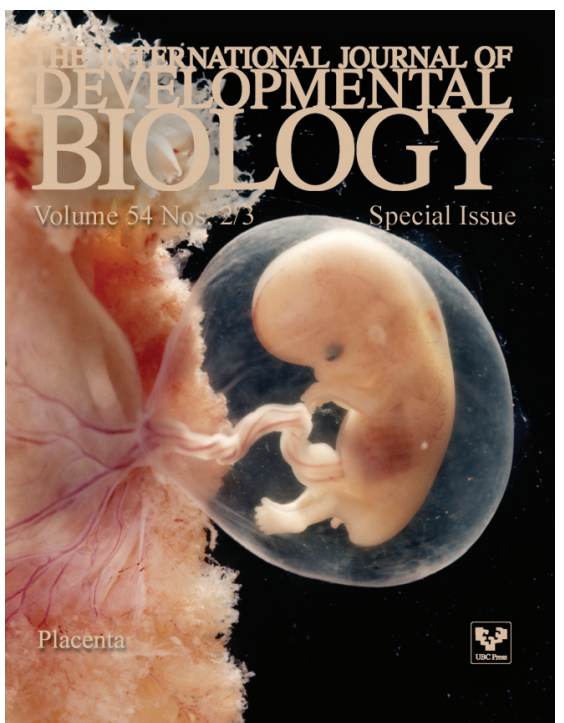

